\section{Psychosocial and Mental Health}

\section{RF-130 WORKPLACE PSYCHOSOCIAL HAZARDS ARE ASSOCIATED WITH EMOTIONAL DISTRESS AND BURNOUT IN CIVIL AVIATION PILOTS}

${ }^{1}$ Chung-Yen Chen. ${ }^{1}$ National Taiwan University, Taiwan

10.1136/OEM-2021-EPI.376

Introduction Pilots have been deemed as safety-sensitive personnel, and they face work patterns of long working hours and jet lag. Failure to properly recover from fatigue may cause adverse health effects, affecting flying public flying safety.

Objectives This study explores the distribution of national civil aviation pilots' work stress and its association with health impacts.

Methods In cooperation with the Taoyuan Union of Pilot, we had issued structured online questionnaires to member pilots as part of the survey. The questionnaire records the respondent's seniority, flight time, and flight characteristics. We assessed workplace psychosocial hazards with the Chinese version of the Job Content Questionnaire (C-JCQ). Mental health conditions were measured with the Brief Symptom Rating Scale (BSRS-5), sleep status with the Epworth Sleepiness Scale (ESS), and burnout with the Chinese version of the Copenhagen Burnout Inventory (C-CBI). Fatigue symptoms on duty were also recorded. The correlation between workplace psychosocial hazards and health effects were analyzed by the regression model.

Results 160 pilots had filled out the questionnaire, with an average age of 40 , ten years of work experience, and an average flight time of 753.3 hours per year. The standardized scores for work demand and work control were 57.8 and 54.7, respectively, higher than the national norm. $28.76 \%$ of pilots had emotional distress. Daytime sleepiness was observed among $23.13 \%$ of pilots. $25.63 \%$ of pilots had work-related burnout with moderate or higher severity. High work demand, low work control, and numbers of red-eye flights were predictors of emotional distress and work-related burnout, and the average flight time is also significantly positively correlated with work-related burnout.

Conclusion Characteristics of active work with high work demand and high work control were observed in national civil aviation pilots. A considerable proportion of pilots experience emotional distress, daytime sleepiness, and burnout.

\section{RF-232 THE POPULATION MAJOR DEPRESSIVE DISORDER IN THE YEARS AFTER SEVERE WILDFIRES IN PORTUGAL CENTRO REGION: THE ROLE OF PERCEIVED HEALTH STATUS, HEALTH VULNERABILITY, RESILIENCE AND SENSE OF COHERENCE}

${ }^{1}$ Mariana Neto, Sónia Namorado, João Pedro Pimentel, António Morais. 'National Institute of Health Dr. Ricardo Jorge, Portugal

\subsection{6/OEM-2021-EPI.377}

Objective The aim of this study was to identify the effects of severe wildfires on the population's mental health in the long term and the role of perceived health status, health vulnerability, resiliency and sense of coherence.
Methods A longitudinal survey with 3 waves (9, 19 and 24 months after the wildfire) was carried out in a representative sample of the municipalities'population affected by the 2017 severe wildfires in the central region of Portugal with data collection by questionnaire on CAPI mode. PHQ 8 (major depressive disorder-MDD), sense of coherence (SOC) and resiliency (CD-RISC) scales were included along with 4 questions related to perceived health status and demographic variables. Statistical analysis was performed using generalized estimating equations approach.

Results 312 individuals (95\% of the sample) participated in the set of the 3 waves. The prevalence of MDD was higher than the overall value of the country $(9.1 \%)$ and of the region $(9.75 \%)$ with higher values in the 1 st and $3 \mathrm{rd}$ waves (21\% and $20 \%$, respectively) and a decrease in the 2 nd wave (11.6\%). No significant relation between resiliency and MDD over time was observed, but SOC, perceived health status and the expected health worsening showed a negative relation with MDD, except for individual disease vulnerability.

Conclusion The higher prevalence of MDD 24 months after wildfires exposure is a great concern. The influence over time of perceived health status and expected health worsening points to the need for a more integrated approach of mental and GP care. The SOC influence shows that participants still have personal resources to face adversity but personal disease vulnerability along with the lack of resiliency effect suggests a low adaptation ability in this population that should be enhanced.

\section{RF-267 AGE AND GENDER DIFFERENCES IN PERCEIVED WORK- RELATED MENTAL HEALTH IN NEW ZEALAND}

${ }^{1}$ Trang Khieu. 'WorkSafe New Zealand, New Zealand

\subsection{6/OEM-2021-EPI.378}

Introduction Work-related mental health has increased considerably and become a major health and safety concern in New Zealand. It could affect work performance and productivity, and lead to a number of serious work-related injury and other illnesses. This study aims to explore impact of age and gender on self-reported work-related mental health among workers across all industries in New Zealand.

Methods Over 4,000 participants were recruited to the survey. Both online and telephone interviews were undertaken. A small number of face-to-face interviews were taken in high deprivation areas. Weighting age by gender within industry was applied to prevent bias and ensure the population representativeness.

Results Overall, 62\% of workers mentioned experiencing at least one of work-related mental health issues (either depression or anxiety or stress) within 12 months prior the study time. The prevalence of work-related stress, anxiety and depression reported by workers was 59.8\%, 31.2\% and $19.7 \%$, respectively. Work-related mental illnesses were reported statistically more prevalently in females compared to males $(64.3 \%$ vs. $59.8 \%)$. Elderly workers aged 60 years and above were statistically less likely to report work-related mental health issues compared to other age groups. When stratified gender by age group, young female workers from 18 to 29 years of age were statistically more likely to report experiencing work-related mental health issues than any other groups of workers. 
Conclusion Perceived work-related mental health is greatly affected by age and gender. Improving mentally healthy working environment could be more effective if workers' age and gender are taken into account. Future studies are needed to identify any difference of demographic characteristics between self-reported work-mental health issues by workers and diagnosed mental illness resulting from work by doctors for better workplace health and safety interventions.

\section{RF-270 DOES THE TIMING AND DURATION OF MENTAL HEALTH PROBLEMS DURING CHILDHOOD AND ADOLESCENCE MATTER FOR LABOUR MARKET PARTICIPATION OF YOUNG ADULTS?}

${ }^{1}$ Samira de Groot. 'University of Groningen - University Medical Centre Groningen, Netherlands

\subsection{6/OEM-2021-EPI.379}

Introduction Little is known about the effect of timing and duration of mental health problems (MHPs) on young adults' labour market participation (LMP).

Objective This life-course study aims to examine whether and how the timing and duration of MHPs between ages 11 to 22 are associated with LMP at age 26.

Methods Data was used from Tracking Adolescents' Individual Lives Survey (TRAILS), an ongoing Dutch prospective cohort study. Internalising and externalising problems were measured by the Youth/Adult Self-Report at ages 11, 13, 16, 19 and 22. LMP (having a paid job yes/no) was assessed at age 26. Logistic regression analyses were performed with 15 -year follow-up data $(\mathrm{N}=874)$.

Results Internalising problems at all ages and externalising problems at ages 13,19 and 22 were associated with an increased risk of not having a paid job (internalising problems odds ratios [ORs] ranged from 2.24, 95\% confidence interval [CI] $1.02-4.90$ at age 11 to OR 6.58 , CI $3.14-13.80$ at age 22 ; externalising problems ORs from 2.84, CI 1.11-7.27 at age 13 to OR 6.36, CI 2.30-17.56 at age 22). Especially young adults with a long duration of internalising problems had an increased risk of not having a paid job in young adulthood compared with participants without mental health problems (OR 3.20, CI 1.88-5.45).

Conclusion Regardless of the timing, experiencing MHPs during childhood or adolescence increases the risk of not having a paid job. In particular, those young adults with a long duration of internalising problems are at risk of not having a paid job. These findings emphasize the necessity of applying a lifecourse perspective when investigating the effect of MHPs on LMP. Early monitoring, mental health care, and timely provision of employment support may improve young adult's participation in the labour market.

\section{RF-382 ASSOCIATION BETWEEN WORKPLACE BULLYING AND ACUTE AND CHRONIC LOW BACK PAIN IN CIVIL SERVANTS FROM A MIDDLE-INCOME COUNTRY}

${ }^{1}$ Fernando Feijó, Neil Pearce, Neice Faria, Maitê Carvalho, Ana Laura Szortyka, Paulo Oliveira, Anaclaudia Fassa. 'Federal University of Bahia, Brazil
Introduction Psychosocial factors at work are risk factors for several health outcomes, including musculoskeletal disorders such as low back pain (LBP). Few studies from high-income countries suggest a positive association between bullying at work and musculoskeletal pain, but the knowledge on this relationship is scarce. None studies on the theme are available from low- and middle-income countries.

Objectives Therefore, we aimed to investigate the association between workplace bullying and LBP in the last 7 days and chronic LBP in civil servants from a middle-income country.

Methods This is a cross-sectional study with 894 participants from judicial civil servants from a city in southern Brazil. Workplace Bullying was measured by the Negative Acts Questionnaire (NAQ-r) and Low Back Pain by the Nordic Questionnaire for Musculoskeletal Symptoms (NQMS). Logistic Regression was used to analyse data and test hypotheses, estimating prevalence odds ratios (POR).

Results The prevalence of workplace bullying was $18.2 \%$. The overall prevalence of LBP in the last 7 days was $50.1 \%$, and of Chronic LBP was $19.3 \%$. Psychosocial factors at work such as occupational stress were strongly associated with both outcomes. Workplace bullying was strongly associated with LBP, even after adjustment for several confounders. The risk of LBP in the last 7 days among bullied workers was $89.0 \%$ higher $(\mathrm{POR}=1.89$; 95\% $\mathrm{CI}$ : 1.31-2.71), compared to non-bullied employees. Bullying was also associated with chronic LBP after adjustment for sociodemographic, behavioural and some occupational factors $(\mathrm{POR}=1.60 ; 95 \% \mathrm{CI}$ : 1.05-2.44).

Conclusions Psychosocial factors at work, particularly workplace bullying, were strong risk factors for LBP, in contrast to most individual factors. Dose-response patterns were showed. Positive associations between bullying and LBP raise hypotheses on causation, emphasising the role of bullying as an important psychosocial risk at work. Further longitudinal studies should address these hypotheses, investigating mechanisms, effect modification and possible mediation.

\section{Respiratory}

\section{RF-112 ASBESTOS-RELATED DISEASE IN THE ONTARIO ASBESTOS WORKERS REGISTER}

${ }^{1}$ Victoria H Arrandale, Paul Demers, Colin Berriault, Leon Genesove, Nathan DeBono, Chaojie Song. 'University of Toronto, Canada

\subsection{6/OEM-2021-EPI.381}

Objectives Research on asbestos-related disease (ARD) has traditionally focused on groups with well-recognized, consistently high exposure. Contemporary exposure to asbestos occurs more intermittently, for example during maintenance of asbestos-containing materials (ACM). The Ontario Asbestos Workers Register (AWR) was established in 1986 to track asbestos exposure. This study reports on the risk of ARD among workers in the AWR.

Methods Overall 33,490 AWR registrants were included. Data were linked probabilistically with administrative health databases (1986-2018) to identify cases of ARD. Workers were followed until diagnosis, death, age 100, last contact with the health system, or end of study, whichever occurred first. Incidence rates were compared to the general population using standardized incidence ratios (SIRs). Associations between 